\title{
TCP performance experiment on LOBS network testbed
}

\author{
Wei Zhang, Jian Wu, Jintong Lin, Wang Minxue, Shi Jindan \\ Key Laboratory of Optical Communication \& Lightwave Technologies, Ministry of \\ Education \\ Beijing University of Posts and Telecommunications, Beijing 100876, P.R.China \\ covery zhang@hotmail.com
}

\begin{abstract}
An LOBS testbed is briefly introduced and TCP performance over OBS is experimentally investigated. Simulation and experimental results show that TCP performance is displayed differently with the loss of ACK (TCP Acknowledgement) burst and data burst.
\end{abstract}

Keywords: OBS, TCP, Data Burst, ACK Burst, Loss Probability

\section{Introduction}

Labeled Optical Burst Switching (LOBS) [1], combining the best of multi-protocol label switching and optical burst switching, has received an increasing amount of attention from both academia and industry worldwide. As the main part of current Internet traffics, TCP performance is directly relative to the performance of whole network. Moreover, it is supposed that TCP will still play a dominant role in the future IP over WDM network, thus it is very important to investigate the behavior of TCP flows over OBS network.

Up to now, most researches about TCP performance over OBS concern the influence of TCP data burst loss on network throughput and the ACK signal link is always supposed to be lossless, which is still far from the actual situation. So it is important to study the influence of ACK burst loss on network performance comparing with data burst loss[2][3][4].

In this paper, we present an LOBS testbed and TCP performance over OBS is investigated both by simulation and experiment [5][6]. Individual experiments of data burst loss and ACK burst loss are carried out to test the influence on OBS network performance. Experimental results show that at the same burst loss probability, the loss of data burst result in worse network performance comparing with ACK burst loss. To lowering the bad influence form traffic contention when network is busy, attaching higher QoS level to data burst traffic is also contributive to the improvement of TCP performance. 


\section{LOBS Testbed Architecture}

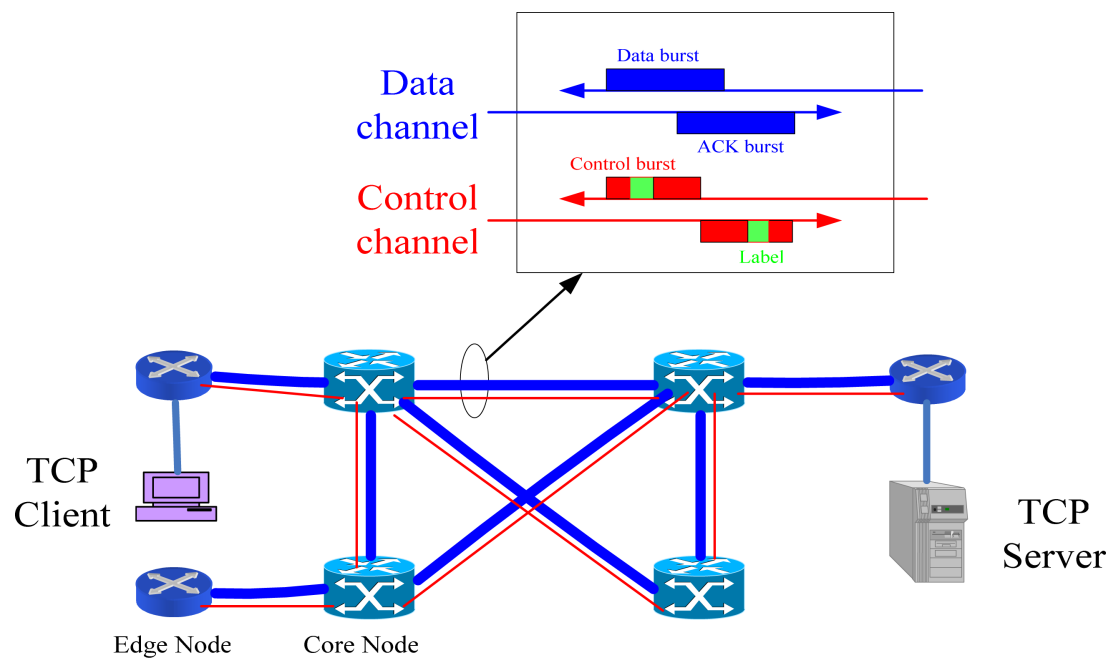

F1g.1. Experimental setup in LUBs testbea.

The LOBS testbed is upgraded based on [1] and enhanced for many aspects. As shown in Fig.1, the testbed is a mesh network comprising three edge nodes (EN) and four core nodes $(\mathrm{CN})$. Each fiber link carries 2 data channels and 1 dedicated control channel, and each channel is $1.25 \mathrm{Gbps}$. At present, each EN supports $4 \mathrm{GE}$ and $8 \mathrm{FE}$ ports to access IP traffic with various QoS demands. In the CN, 4X4 switching matrix, switching speed less than 100ns and processing time less than 10 micro seconds are achieved. Comparing with [1], EN improves the processing capability and applies more flexible algorithms both in assembling and scheduling so that it can combine MPLS mechanism to assembling and support wider range for burst length. In the CN, a much more simple switching architecture is applied to the optical cross-connect providing stable and cost-effective performance. Powerful control plane is constructed to perform routing, signaling and network monitoring function. Both EN and $\mathrm{CN}$ is expandable for further research and the total cost of the system is much lower than the first one. 


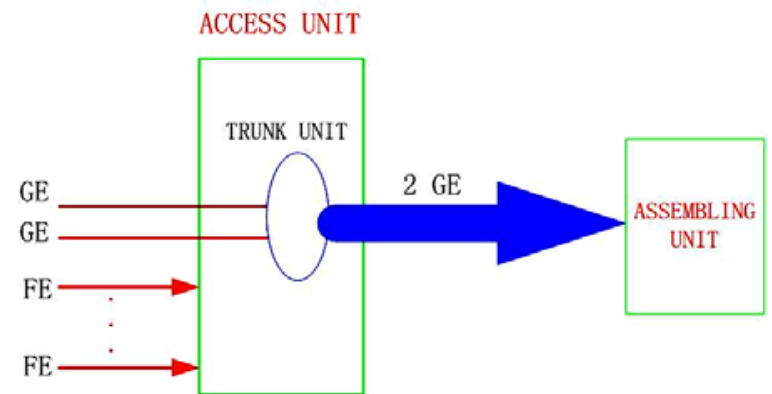

(a) Trunk architecture in EN

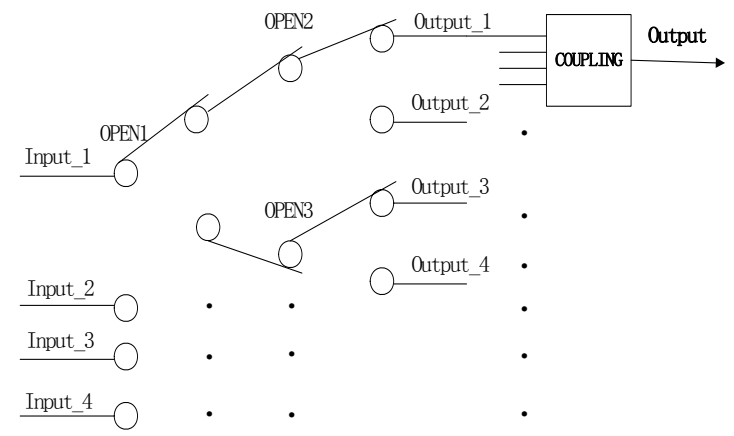

(b) Optical cross-connect architecture in $\mathrm{CN}$

Fig.2. Node specialties in LOBS testbed.

\section{Experimental Setup}

The experiment setup is shown as Fig. 1 and the simulation scenario is set similarly. The system is constructed by four core nodes and three edge nodes. Standard interface such as Fast Ethernet and Gigabit Ethernet are provided to access IP-based traffic. And input packets classified according to their ingress node IP and MAC address to form the FEC (Forward Equivalence Class) queue, based on which data burst is assembled. Meanwhile, the corresponding label is inserted to the control burst. Assembled burst is sent into LOBS network following a corresponding control burst for an offset time. In this experimental setup, burst assembly algorithm with fixed time-1ms and length threshold is applied, and for burst scheduling algorithm latest available unused channel with void fill (LAUC-VF) is used, each with a maximal delay of 2.5 us and 10 us.

As shown, a TCP server is connected to one edge node and the client is connected to the other one, by which a TCP connection can be set up through the LOBS network. Because there are two individual links for each TCP data flow, different burst loss probabilities for data burst and ACK burst can be achieved by configuring these links. In the TCP transmission, a maximum congestion control window of 64KBytes and maximum segment size of 512Bytes are employed in the TCP server. No background 
traffic is added for current experiment and the bursts are dropped deliberately by introducing a specific dropping mechanism to achieve certain burst loss probability.

\section{Simulation, experiment and discussions}

\subsection{Simulation results}

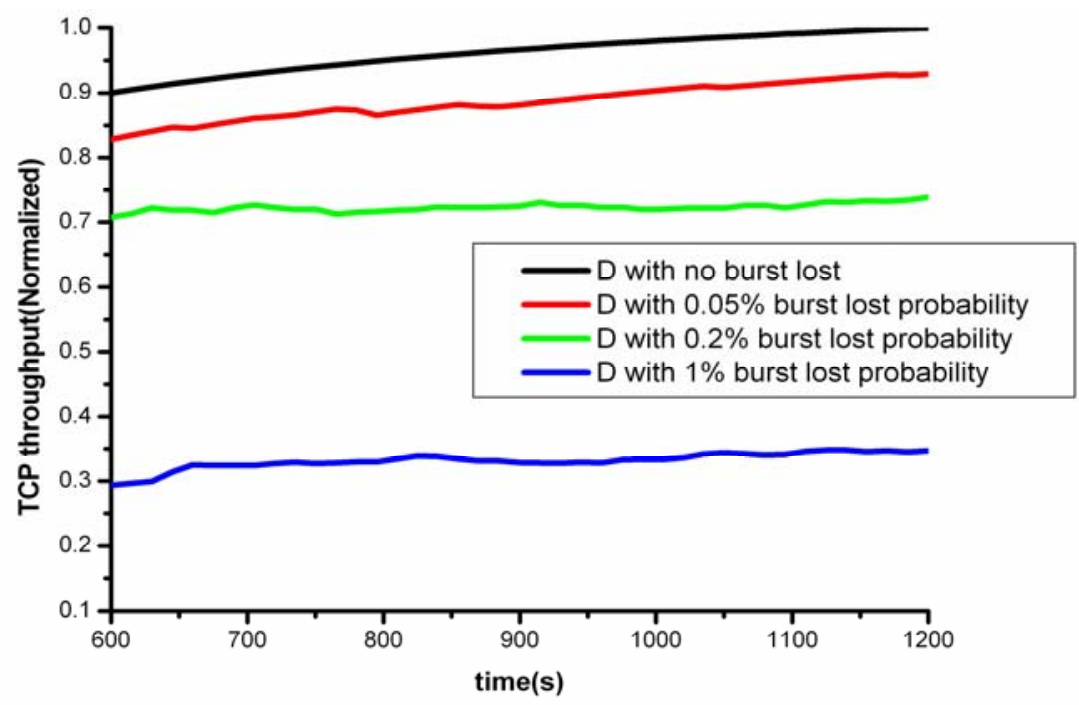

(a) Data burst lost

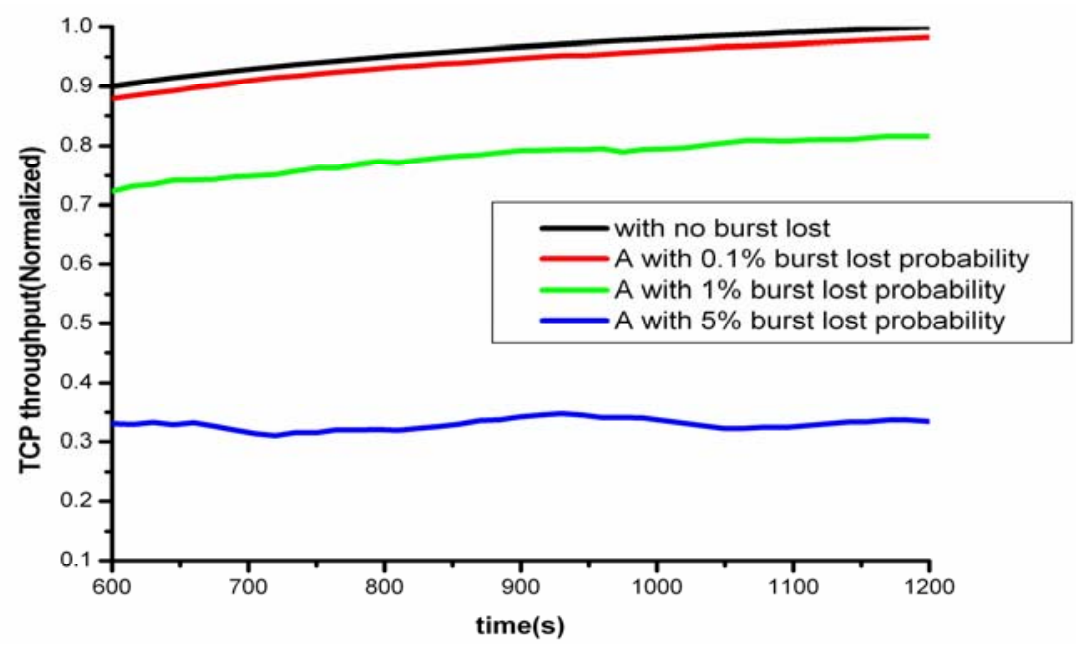

(b) ACK burst lost

Fig.3. Simulation results of TCP throughput in OBS network 
In the simulation scenario, the data burst Dropping Probability (DP) varies from 0 to $1 \%$, as it is shown in Fig[3]a, with increasing dropping probability, TCP throughput decrease accordingly. When the DP is set to $1 \%$, TCP throughput is only $30 \%$ of 0 DP, which shows very bad network performance. The ACK burst lost transmission is shown in Fig[3]b, the TCP throughput is also dropping as the DP increase, but as the DP is set to $1 \%$, the TCP throughput is about $73 \%$ of $0 \mathrm{DP}$, which is much higher than the situation of data burst loss. If we want to get the same results of $1 \%$ DP from data burst, the DP of ACK burst must set to 5\%. It is shown from those results that the influence of ACK burst loss on TCP throughput is much lower compared with data burst loss.

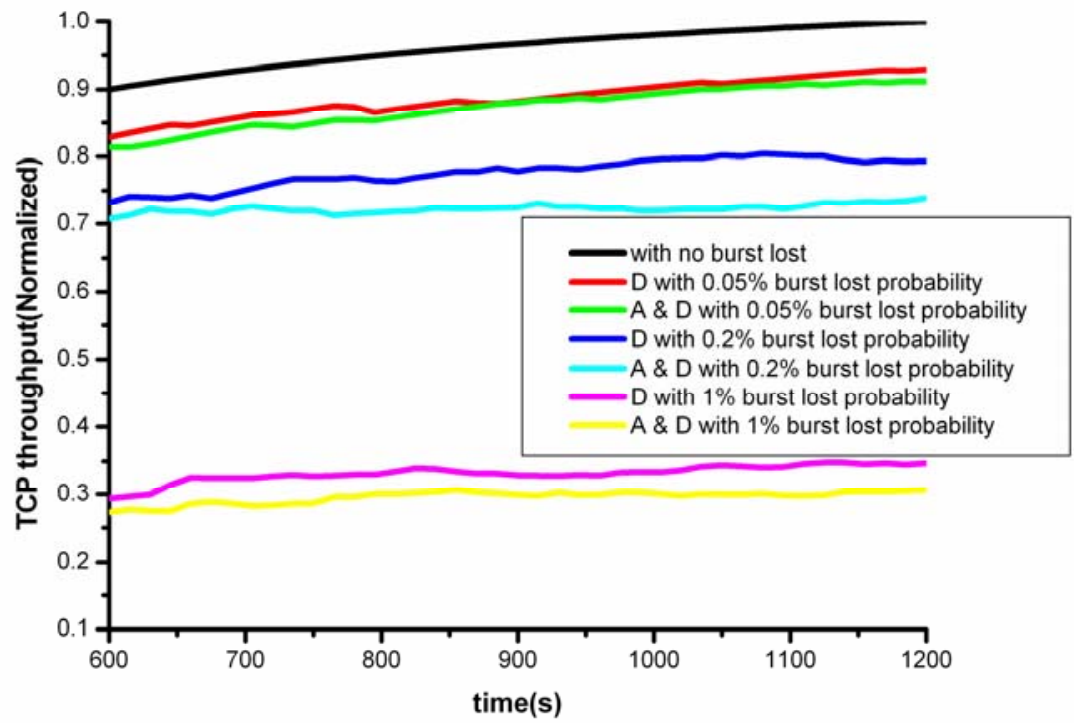

Fig.4. Simulation results of TCP throughput in OBS network, both ACK and data are lost

The case of both ACK and data are lost is shown in Fig[4], it can be investigated from the figure that the loss of ACK burst introduced much lower decrease in TCP throughput.

\subsection{Experimental results and discussions}

In the experiment scenario, FTP server and client are used to transmit TCP traffic. As shown in Fig[1], ACK burst and data burst can be dropped separately. The results are shown as following: 


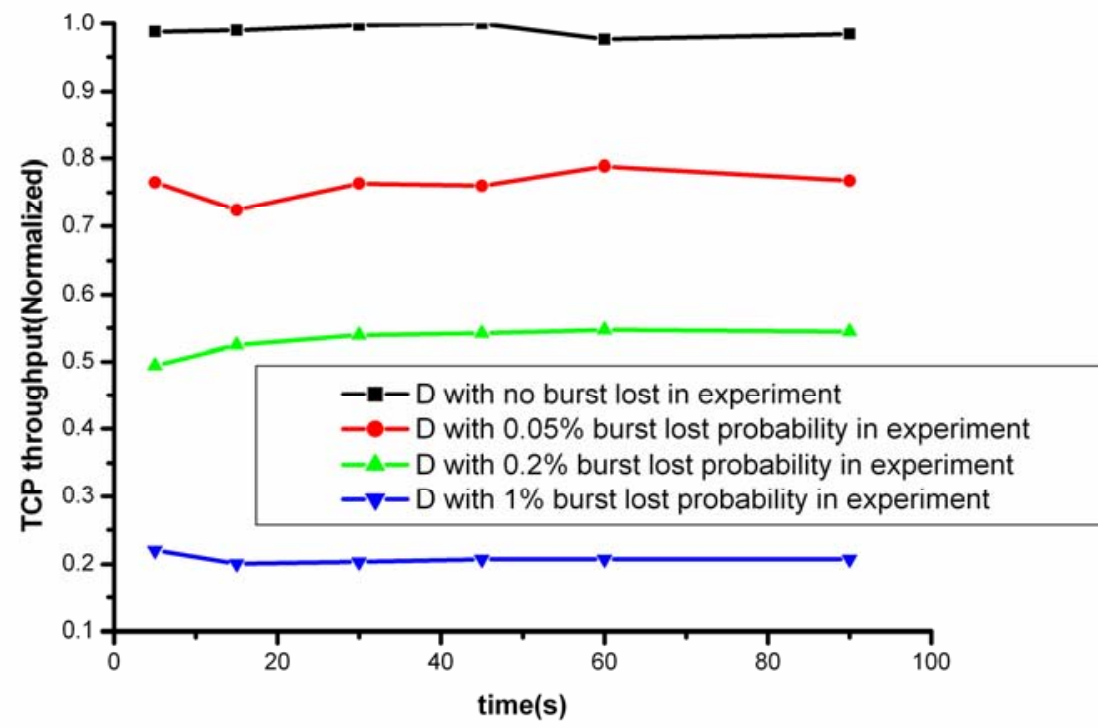

(a) Data burst lost

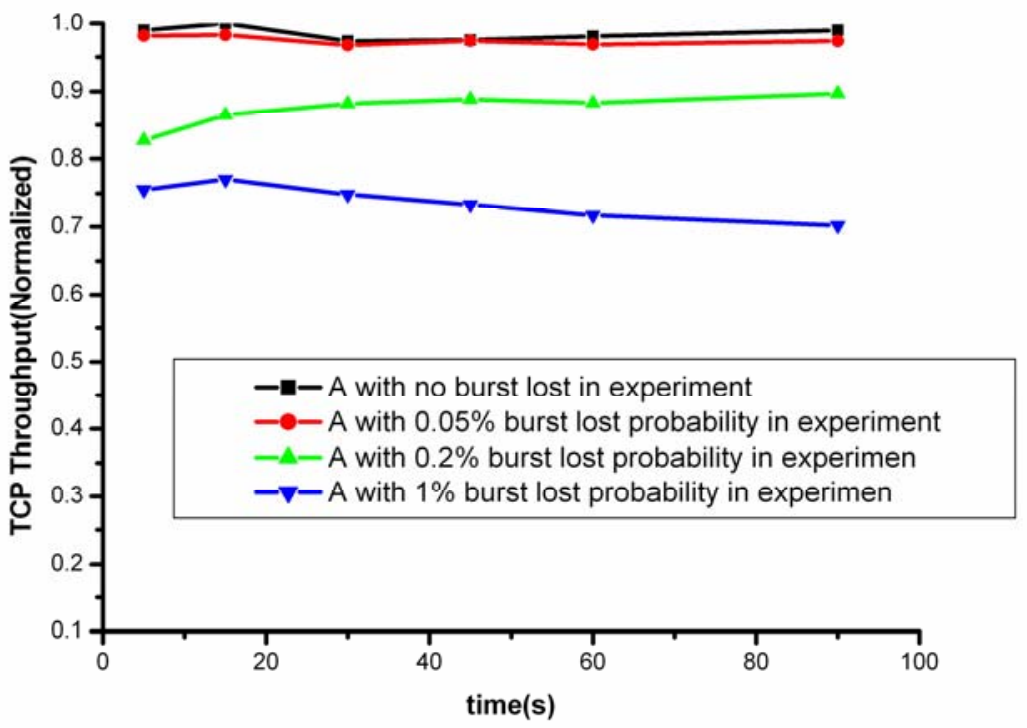

(b) ACK burst lost 


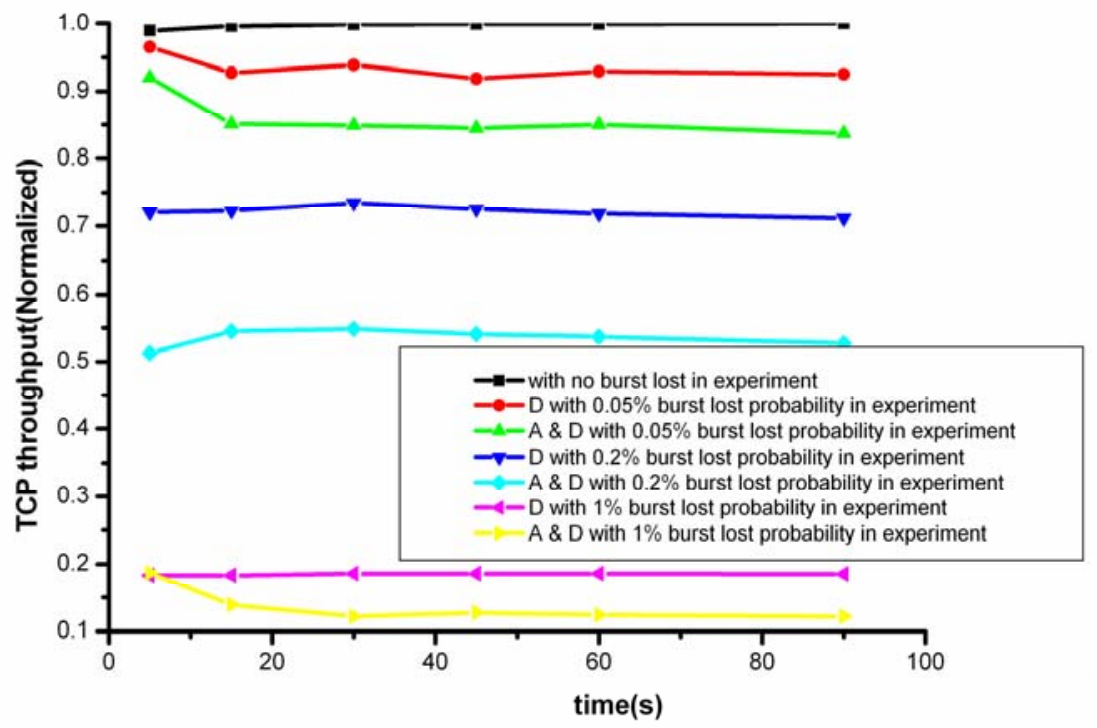

(c) Both ACK and data lost

Fig.5. Experiment results of TCP throughput in OBS network

Similar results from experiments is shown in Fig[5]. The reason for the result that ACK burst loss affect less than data burst loss on TCP throughput performance can be explained by TCP slide Window control mechanism. As we can see from Fig[6], Client A sends data to Client B base on the slide Window control mechanism. Suppose the WIN $=400 \mathrm{~B}$,Length $=100 \mathrm{~B} /$ segament, initial number is 1 . When $\mathrm{B}$ has received all the data sent by $\mathrm{A}$, Client $\mathrm{A}$ will continue sending data to $\mathrm{B}$, though missed a ACK signal. Therefore, if the data transmit with no loss probability, low lost probability of ACK signal will hardly affect the network throughput.

Considering that QoS provision is provided in OBS network[7], we can apply higher class of QoS to data burst and drop the ACK burst in contention resolution while maintaining the network performance to a comparatively stable level. 


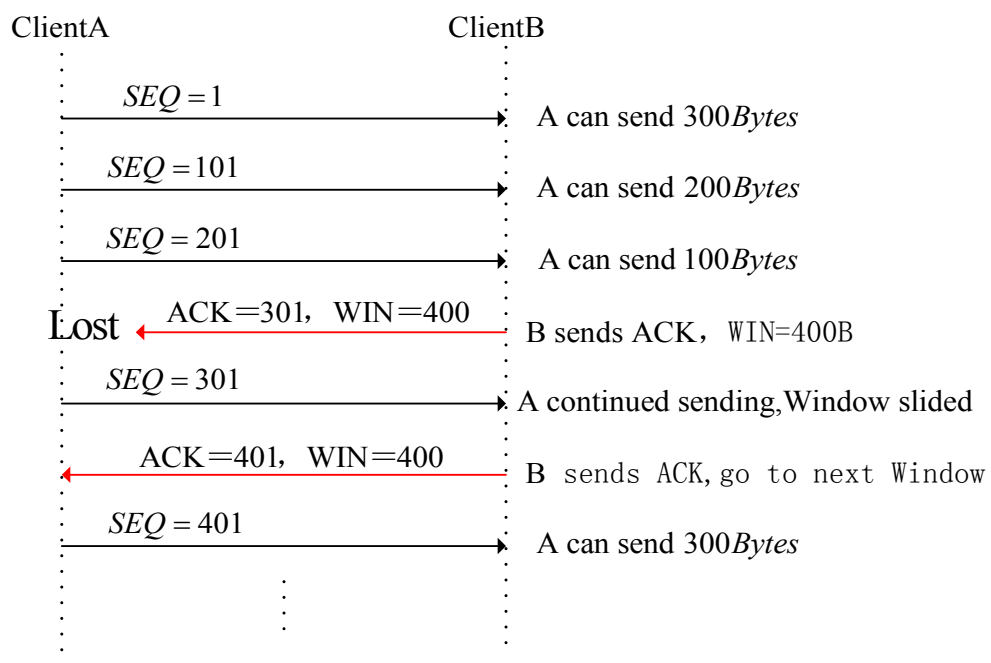

Fig.6. TCP slide Window control mechanism

\section{Conclusions}

In this paper, the TCP performance over OBS is experimentally investigated on OBS testbed [5][6]. Experimental results show that the performance of TCP traffic is influenced in the case of burst loss, both for ACK burst and data burst. But ACK burst loss affect less than data burst loss on TCP throughput performance, caused by TCP slide Window control mechanism. Moreover, considering that QoS provision is provided in OBS network, contention resolution mechanism of dropping ACK burst when network is busy is proposed in further investigations.

\section{References:}

[1] C. Qiao and M. Yoo, "Optical burst switching (OBS) - A new paradigm for an optical internet," J. High Speed Networks, vol. 8, no. 1, pp. 68-84, 1999.

[2] Sunil Gowda, Ramakrishna K Shenai , Krishna MSivalingam, Hakki Candan Cankaya, "Performance Evaluation of TCP over Optical Burst-Switched (OBS) WDM Networks," IEEE ICC 2003

[3] A. Detti and M. Listanti, "Impact of Segments Aggregation on TCP Reno Flows in Optical Burst Switching Networks,” Proceedings IEEE, INFOCOM 2002, New York, NY, June 2002

[4] Robert Pleich, "Performance of TCP over Optical Burst Switching Networks", ECOC'05.September 2005.

[5] Guo H., “A Testbed for Optical Burst Switching Network,” OFC 2005, March 2005.OFC2005

[6] Guo H., "Multi-QoS Traffic Transmission Experiments on OBS Network Testbed,"

ECOC'05.September 2005

[7] Zhang W., Wu J., Xu K. and Lin J.T. "TCP performance experiment on OBS network testbed", OFC2006, March 2006. 